Original Research Paper

\title{
Novel Biomarkers in Determining Prostate Cancer Diagnosis and Prognosis
}

\author{
${ }^{1}$ Zuzana Varchulova Novakova, ${ }^{2}$ Katarina Bevizova and ${ }^{1}$ Iveta Gasparova \\ ${ }^{1}$ Institute of Medical Biology, Genetics and Clinical Genetics, Slovakia \\ ${ }^{2}$ Institute of Anatomy, Faculty of Medicine, Comenius University, Bratislava, Slovakia
}

Article history

Received: 21-07-2014

Revised: 13-09-2014

Accepted: 08-01-2015

Corresponding Author: Zuzana

Varchulova Novakova

Institute of Medical Biology,

Genetics and Clinical Genetics,

Faculty of Medicine,

Comenius University,

Bratislava, Slovakia

E-mail: zuzana.varchulova@fmed.uniba.sk

\begin{abstract}
The clinical behavior and molecular pathology of prostate cancer is highly variable. Current "traditional" prognostic markers cannot reliably distinguish the potentially life-threatening cancer from indolent cancer. Identification of additional new predictors of cancer aggressiveness is therefore urgently required. This communication is aimed at a brief review of new biomarkers in prostate cancer diagnostics and prognostics. Pubmed systematic search was performed to collect both original and review articles addressing prostate cancer prognostic biomarkers using key words genetics, prostate cancer, biomarkers and prognosis. The development of molecular and immunohistochemical methods enabled the identification of potential biomarkers in relation to diagnosis and prognosis. Numerous promising markers and approaches have been identified. Some of these markers may be translated into clinical practice after verification in larger prospective trials in future and can help to determine diagnosis and prognosis of $\mathrm{CaP}$ more accurately.
\end{abstract}

Keywords: Prostate Cancer, Biomarkers, Diagnosis, Prognosis

\section{Introduction}

Prostate Cancer (PCa) is most common nondermatologic malignancy of men in Western Europe (Jemal et al., 2009; Ziaran et al., 2009). The clinical behavior and molecular pathology of $\mathrm{PCa}$ is highly variable. Identifying patient subgroups that require less treatment from those that should be targeted with more aggressive therapy is therefore a key goal. Because of the highly variable natural history of $\mathrm{PCa}$, additional new predictors of cancer aggressiveness are urgently required. Over-treatment of PCs is a particular concern leading to substantial cardiovascular and skeletal morbidity (Ziaran et al., 2013a; 2013b). This is especially true for many Prostrate-Specific Antigen (PSA) screen-detected cancers, which in the absence of treatment, may never become life threatening. Conversely, more conservative approaches to disease detection and management can leave potentially aggressive cancers untreated. Therefore, improved biomarkers are required to allow radical therapies to be targeted to men with potentially lethal cancers, so that the others, with more benign-behaving indolent cancers, are spared inappropriate treatment.

Current "traditional" clinicopathologic prognostic markers predictive of outcome in men with $\mathrm{CaP}$ after Radical Retropubic Prostatectomy (RRP) consist of
Gleason score, TNM stage, surgical margin status and preoperative serum Prostate-Specific Antigen (PSA) (Zummerova et al., 2010; Epstein et al., 2005; Repiska et al., 2005). Beyond the current clinicopathologic parameters, there have been other biomarkers and approaches proposed to: (i) Distinguish between indolent and potentially life threatening disease (ii) aid the decision for rebiopsy in previous negative biopsies with rising PSA, (iii) monitor the disease progression and its responsivness to therapy These approaches and markers include GenomeWide Association Studies (GWAS), chromosomal aberrations, DNA-based markers, RNA-based biomarkers and protein markers (tissue, serum, urine biomarkers) (Manolio, 2010). Available methods to identify potential biomarkers include genomics, proteomics and tissue based immunohistochemical staining. Quantitation of cancer biomarker transcripts using Real-Time quantitative Polymerase Chain Reaction (qRT-PCR) of large samples may help in the search for clinically useful cancer biomarkers that can be integrated into clinical trial design (Jiang et al., 2007). Gene expression array technology applied to PCa has resulted in the identification of a number of genes that have been associated with outcome. More recently, Next-Generation Sequencing (NGS) have been described, which could bring promising information in 
our understanding of the cancer genome of several tumor types (Macconaill and Garraway, 2010).

This article provides review of current most promising molecular and immunohistochemical biomarkers in $\mathrm{CaP}$ diagnosis, prognosis and clinical behavior.

\section{Materials and Methods}

Pubmed systematic search was performed to collect both original and review articles addressing $\mathrm{CaP}$ prognostic biomarkers using key words genetics, prostate cancer, biomarkers and prognosis.

\section{Results and Discussion}

\section{Genetics of Prostate Cancer}

Initiation of prostate cancer is a process resulting from the progressive accumulation of genetic disorders. On the basis of the constellation of polymorphisms of germ cell cancer the risk of developing cancer is individual. The cumulative effect of polymorphism genome may lead to one or more disorders and/or may "offer" an environment for further genetic alterations in prostate epithelial cells. Over time, additional somatic genetic disorders alter the behavior of prostate epithelial cells until it represents signs of malignancy (Zheng et al., 2008). Epigenetic alterations are other common events in carcinogenesis, including $\mathrm{CaP}$, which may lead to aberrant expression of critical genes such as tumor suppressors and oncogenes. Although most $\mathrm{CaP}$ are classified into a single group of adenocarcinomas, there is no universal molecular path of $\mathrm{CaP}$ development. It is also unlikely that two prostate adenocarcinomas share the same genetic path in the development of cancer. Genetic and genomic technologies have helped to clarify the changes in genes that lead to the development of $\mathrm{CaP}$. These changes provide a molecular basis from which diagnostic, prognostic and predictive biomarkers can be developed (Febbo, 2009). For genetically determined diseases, genetic alterations can be identified via methods of molecular genetics (e.g., polymerase chain reaction, fluorescence in situ hybridisation, genome sequention). These alterations, when identified, can be in turn used as biomarkers for prognosis of $\mathrm{CaP}$ and other cancers.

\section{Criteria for a Candidate Biomarker}

The National Cancer Institute defines a biomarker as a biological molecule found in blood, other body fluids, or tissues that is a sign of a normal or abnormal process or of a condition or disease. A biomarker may be objectively measured and evaluated as an indication of normal biologic processes, pathogenic processes, or pharmacologic responses to a particular treatment or condition.
Biomarker is an analyte that signifies the presence or degree of a biological process, which in itself is frequently directly linked to the clinical expressions and result of a particular disease. The selection of a cancer biomarker should have a biological or therapeutic basis or, at minimum, the biomarker should indicate a reliable correlation with the presence, characteristics, or aggressiveness of the cancer. Also, there should be an evaluation of the strength of the marker in relation to the outcome of the disease, which, together with other factors, should be carried out as an independent predictor in a multivariable assay in the general population (Hartwell et al., 2006). Biomarkers for the diagnosis and prognosis of PCa include DNA-based markers, RNAbased biomarkers and protein markers. They may be useful for prognostic purposes in the outcome of diseases, with particular attention on the quantitative biomarkers that demonstrate a relationship with the clinical manifestation of the disease and that have an effect on quality of life, risk of complications, or survival. Surrogate biomarkers have a significant function in disease monitoring after accepted treatments are introduced. Surrogates are particularly important for those treatments that are uncommon, such as cases in which the direct study has proved to be very difficult because of the limited number of patients and varying expression of their primary illness or in which the efficiency of the treatment must justify the high cost.

\section{Identifying Discriminating Markers}

With the completion of the Human Genome Project, the publication of the International Haplotype Map Project (a catalog of millions of common single nucleotide polymorphisms, or SNPs, in the human population) and a decrease in the cost of high-throughput genotyping, an unbiased genomewide search for inherited variants associated with $\mathrm{PCa}$ risk has become feasible. This approach, called a Genome-Wide Association Study (GWAS), scans the entire genome, evaluating common inherited variants (minor allele frequency $>1-5 \%$ in the population) in large numbers of cases and controls (Manolio, 2010).

GWAS indicate genetic heterogeneity for the onset of disease with numerous low risk loci described along with two notable high-risk loci at $8 \mathrm{q} 24$ and $7 \mathrm{q} 31$. The linked loci on $8 \mathrm{q} 24$ are located immediately downstream of the MYC gene that is upregulated in PCa (Beuten et al., 2009; Robbins et al., 2007).

There are several potential mechanisms by which a genetic variant may be associated with altered cancer risk, including: (i) Genetic linkage to a coding variant in a cancer-relevant gene (i.e., the risk SNP is merely a proxy for the true causal exonic variant that was not tested in the GWAS), (ii) alteration in promoter/enhancer binding sites or chromatin structure affecting expression 
of adjacent or distant genes, or (iii) change in the expression of noncoding RNAs. There is also a high probability that $\mathrm{PCa}$ genes/alleles act cooperatively in the aetiopathogenesis of the disease supporting the notion that it is unlikely that any one biomarker alone is likely to be conclusive in detecting and predicting outcome of cancer (Clarke et al., 2010).

\section{Novel Biomarkers for CaP Diagnosis}

\section{The PCA3 and TMPRSS2: ERG Fusion}

PCA3 is a noncoding RNA with expression confined to the prostate and which is highly overexpressed in $95 \%$ of PCas compared with normal or benign hyperplastic prostate tissue (Salagierski and Schalken, 2012). PCA3 has been assayed from urine following prostatic massage in 11 separate clinical studies totalling 2737 men from Western countries (Tosoian et al., 2010; Van Gils et al., 2007; Marks et al., 2007) with an overall sensitivity of $69 \%$ and specificity of $70 \%$ for men with $\mathrm{PCa}$. The role of PCA3 in clinical practice as a commerciallyavailable test remains uncertain with most advocates indicating a place in patients who have already had TRUS biopsies with a negative result for cancer but in whom PCa remains suspected.

Detection of the TMPRSS2: ERG fusion in urine has been reported to yield $>90 \%$ specificity and $94 \%$ positive predictive value for PCa detection (Hessels et al., 2007), although a clinical diagnostic test is not yet available. The combination of urinary PCA3 and TMPRSS2ERG with serum PSA levels has been reported to improve screening performance compared to PSA alone (Salami et al., 2013). Moreover, the recent study indicates that integration of levels TMPRSS2: ERG transcripts in urine, with PCA3-score androgenic status, genetic status and traditional clinical variables could significantly increase detection of high risk localized PCa (Cornu et al., 2013).

\section{Early Prostate Cancer Antigen}

Leman et al. (2007) reported results on a serum biomarker called Early Prostate Cancer Antigen (EPCA) using an antibody assay against the EPCA-2.22 epitope. The study involved 385 men and reported a 92\% specificity for healthy men and men with benign prostatic hyperplasia and a 94\% sensitivity for overall PCa detection. In addition, the authors indicated that EPCA2.22 was highly accurate in differentiating between localized and extracapsular disease (Witt et al., 2000).

\section{SPINK1}

SPINK1 (also referred to as TAT1) is a biomarker for $\mathrm{PCa}$ that can be detected in prostatic massage urine. SPINK1, a trypsin inhibitor secreted from pancreatic acinar cells, is thought to function in the prevention of trypsin-catalyzed premature activation of zymogens within the pancreas and the pancreatic duct. Mutations of this gene are associated with hereditary pancreatitis and tropical calcific pancreatitis (Bhatia et al., 2002).

Laxman et al. (2008) showed that a multiplexed qPCR assay including SPINK1 on sedimented urine from patients presenting for prostate biopsy or prostatectomy outperformed serum PSA or PCA3 alone. SPINK1 expression in urine is also an independent predictor of biochemical recurrence after resection. On the other hand, recent study concludes that SPINK1 protein expression (evaluated by immunochemistry) may not be a predictor of recurrence or lethal PCa amongst men treated by radical prostatectomy (Flavin et al., 2014).

\section{$\alpha$-Methylacyl Coenzyme A Racemase (AMACR)}

AMACR is an enzyme localized to the peroxisome and involved in fat metabolism and has been identified to function as a growth promoter, independent of androgens, in prostate cancer (Zha et al., 2003). By using various experimental methods and different PCa specimens, the AMACR gene has been shown to be overexpressed in PCa tissue at the mRNA and protein levels and making it a highly specific tissue biomarker currently used to aid in the pathological diagnosis (Jiang et al., 2004).

When PCa tissues were compared with normal controls, a 9-fold increase in mRNA levels of AMACR was discovered in $88 \%$ of the sample PCa tissues (Rogers et al., 2004). Immunodetectable serum autoantibodies generated in response to the AMACR tumor-associated antigen may also be useful in preliminary diagnosis, especially if combined with PSA screening. A considerably more enhanced sensitivity and specificity in PCa patients with mid-range PSA levels have been observed with AMACR antibodies than that with PSA. This demonstrates that AMACR can be useful in discriminating control subjects from those with $\mathrm{PCa}$ (Sreekumar et al., 2004). Interestingly, it has been described, that trifluoroibuprofen, an AMACR inhibitor, reduces cancer cell proliferation and Inhibits in vivo tumor growth in aggressive PCa models (Festuccia et al., 2014). This makes AMACR one of possible therapeutical targets in future.

\section{Glutathione S-transferase P1 (GSTP1)}

GSTs are aubiquitous family of multifunctional enzymes that conjugate reactive substrates with reduced Glutathione (GSH) and are involved in detoxification. Their role is in protecting the cells from oxidative attack. The GSTP1 gene has been observed to be unmethylated in all normal human tissues and $\mathrm{BPH}$, but hypermethylated in specimens of PCa tissues (Harden et al., 2003). GSTP1 has been shown to be acutely sensitive in detecting the presence of prostatic intraepithelial neoplasia and $\mathrm{PCa}$, 
thereby distinguishing patients with these diseases from patients with BPH (Lee et al., 1994).

\section{Biomarkers for Determining CaP Prognosis and Progression}

\section{LosS of PTEN}

The PTEN gene on $10 \mathrm{q} 23$ is mutated in up to $1 / 3$ of hormone refractory $\mathrm{PCa}$ and homozygous deletions and mutations have been identified in a subset of primary $\mathrm{PCa}$. Loss of PTEN protein in primary $\mathrm{PCa}$, as determined by immunohistochemistry, correlates with high Gleason score and advanced stage (McMenamin et al., 1999). PTEN is a dual protein and lipid phosphatase that is responsible for dephosphorylation and inactivation of phosphatidylinositol 3,4,5-trisphosphate (PIP3), a second messenger that is produced after activation of PIP3 kinase in response to ligation of several growth factor receptors, including IGF-1. PIP3 activates the protein kinase AKT. AKT signalling results in inhibition of apoptosis in response to a variety of signals and to increased cell proliferation (Vivanco and Sawyers, 2002). In assessing the relationship of PTEN deletion with the TMPRSS2-ERG fusion, two independent groups found that patients with neither lesion had a favorable prognosis (Reid et al., 2010).

Other markers tested in combination with PTEN loss for prognostic information include tumor protein p27 gene loss (Halvorsen et al., 2003), hemoxygenase-1 overexpression (Li et al., 2011) and HER2/3 overexpression (Ahmad et al., 2011). A four-protein signature, as assessed by immunohistochemical staining for PTEN in combination with a subset of proteins involved in tumor growth factor-b signaling: SMAD4, cyclin D1 and SPP1, was found to predict biochemical recurrence significantly better than Gleason score alone (Ding et al., 2011). The most promising pathway in which this is likely to be employed in the near future is the PTEN/PI3K pathway as a number of clinical trials using inhibitors of this pathway are in development or underway in PCa (Thomas et al., 2004).

Thus, the measurement of PTEN protein levels and downstream targets of AKT in prostate needle biopsies may have value in the future if these trials show promise (Thomas et al., 2004). It remains to be determined which combinations of events will provide the most reliable prognostic information to guide clinical decision making. Moreover, it has been found that PI3K and Androgen Receptor (AR) pathway crosstalk plays an intortant role in castrate resistant $\mathrm{PCa}$ development, with potentially important implications for PCa etiology and therapy (Mulholland et al., 2011).

\section{$C-M Y C$}

The C-MYC protein is a nuclear transcription factor that regulates a number of cellular processes including cell cycle progression, metabolism, ribosome biogenesis, protein synthesis and mitochondrial function (Dang et al., 2006). In PCa, there is evidence that $\mathrm{C}-\mathrm{MYC}$ is involved in $\mathrm{PCa}$ progression since a region encompassing the MYC locus (8q24) is somatically amplified at low levels in a subset of patients and the presence of amplification in this region correlates with both high histological grade and worse prognosis (Ribeiro et al., 2007).

It has been long known that a subset of PCa lesions express elevated levels of MYC mRNA, often in parallel with increased expression of PIM-1, a gene known to cooperate with MYC in other malignancies (Tomlins et al., 2006) and that is often overexpressed in PCa (Cibull et al., 2006). Targeted overexpression of the human MYC gene in the mouse prostate results in early invasive prostate adenocarcinoma and rare metastatic adenocarcinoma (Ellwood-Yen et al., 2003). These findings provide evidence that C-MYC overexpression can drive neoplastic transformation in the mouse prostate and thus may play a role in initiation and progression of human $\mathrm{PCa}$.

\section{$A Z G P 1$ and $h C A P-D 3$}

Zinc-Alpha2-Glycoprotein (AZGP1) is present in high concentration in human seminal plasma and considered to be a soluble homologue of MHC-I (Hassan et al., 2008). Some studies reported on the highly predictive value of AZGP1 expression after Radical Prostatectomy (RP) specimens as as a predictor of metastatic PCa (Henshall et al., 2006). In addition, urine detected AZGP1 showed promising results in the prediction of $\mathrm{PCa}$, making him a potentional urine biomarker (Katafigiotis et al., 2012). Lapointe et al. (2008) reported a combination of immunohistology for AZGP1 and RNA in situ hybridisation for hCAP-D3 expression in tissues from RP specimens which distinguished even more clearly those patients whose tumours would reccur.

\section{Annexin A3 (ANXA3)}

ANXA3 has an inverse relationship to cancer and the immunhistochemical staining in prostatic tissue correlates with disease progression, Gleason score and malignancy. ANXA3 belongs to a family of calcium and phospholipid binding proteins that are implicated in cell differentiation and migration, immunomodulation, bone formation and mineralization in PCa metastasis (Gerke et al., 2005). ANXA3 represents a promising candidate tissue marker and when combined with the standard prognostic parameters, may provide a more precise prediction of prognosis in the individual patient (Köllermann et al., 2008).

\section{Forkhead Box Protein Al (FOXA1)}

FOXA transcription factors are potent, contextspecific mediators of development that hold specialized 
functions in hormone-dependent tissues. Over the last several years, FOXA1 has emerged as a critical mediator of nuclear steroid receptor signalling, manifest at least in part through regulation of androgen receptor and oestrogen receptor activity. Recent findings point towards a major role for FOXA1 in modulating nuclear steroid receptor activity in breast and $\mathrm{PCa}$ and suggest that FOXA1 may significantly contribute to protumourigenic phenotypes (Augello et al., 2011).

Jain et al. (2011) examined the expression of forkhead box protein A1 (FOXA1). Their findings suggest that increased expression of FOXA 1 is associated with the development of metastatic $\mathrm{CaP}$. Metastatic PCa specimens demonstrated high nuclear FOXA1 staining in $89 \%$ of tissues as compared with $19 \%$ of patient-matched primary tumour samples. FOXA1 colocalized with androgen receptor in all samples and FOXA1 levels were positively correlated with tumour size, extraprostatic extension and lymph node metastasis. Such data implicate that FOXA1 is strongly associated with metastatic disease in PCa.

\section{Epigenetic Alterations and Prostate Carcinogenesis}

Epigenetic alterations represent important contributing factors in prostate carcinogenesis and may provide useful biomarkers for disease progression (Nelson et al., 2009). For example, DNA methylation has been implicated in silencing genes involved in signal transduction, hormonal response, cell cycle control and oxidative damage response, such as GSTP1 and others (Harden et al., 2003).

One key modification associated with prostate carcinogenesis is trimethylation of lysine residue 27 of his tone $\mathrm{H} 3$ (H3K27-me3), which is mediated by the his tone methyltransferase enzyme Ezh2, a key oncogenic driver of advanced disease and metastasis (Varambally et al., 2002). Since the H3K27-me3 mark is associated with transcriptional repression, increased levels in $\mathrm{PCa}$ are associated with repression of tumor suppressor genes such as DAB2IP, a member of the Ras GTPase family (Chen et al., 2005).

A number of other genes have also been found to be hypermethylated in $\mathrm{PCa}$. Using quantitative real-time methylation specific PCR (Real Time-MSP), Yegnasubramanian et al. (2004) assessed the extent of hypermethylation in 16 different genes in $\mathrm{PCa}$ and found strikingly high frequencies of hypermethylation in the $\mathrm{CpG}$ islands associated with APC, RASSF1a, PTGS2 and MDR1, but virtually no methylation in normal prostate tissues.

It is clear that epigenetic regulation plays an important role in the development and progression of $\mathrm{CaP}$, but the significance of identified genes still remains hypothetical (Febbo, 2009). There is mounting evidence, however, that methylation of genes (e.g., GSTP1, APC, PTGS2, EDNRB and T1G1) plays an important role in the development and prognosis of $\mathrm{CaP}$, that makes then promising biomarkers in near future (Tomlins et al., 2005).

\section{Androgen Receptor and Prostate Cancer}

Although androgen deprivation therapy is the gold standard for the treatment of metastatic $\mathrm{CaP}$, patients gradually become resistant to castration levels of androgens and the disease progresses. It is now clear that even though $\mathrm{CaP}$ progression in a state of low levels of androgens, most cancers is still dependent on stimulation of Androgen Receptor (AR) (Ziaran et al., 2011).

In the vast majority of $\mathrm{PCa}$, it is clear that $\mathrm{AR}$ function is essential for tumor development and progression, that these activities are supported by FOXA1 and that resurgent AR activity after hormone therapy (a hallmark of the transition to lethal disease) requires FOXA1 activity (Linja et al., 2001).

At present, it is impossible to distinguish carcinoma, which is really independent of AR stimulation and cancer which maintains the dependence. Therefore, there are currently being developed predictors based on AR transcriptional activity, which could predict the activity of AR during treatment (Pulukuri et al., 2007).

In normal prostate epithelium, AR suppresses cellular proliferation, as probasin-Cre-mediated conditional deletion of $\mathrm{AR}$ leads to increased proliferation accompanied by decreased expression of differentiation markers (Kawamoto et al., 2007). In PCa, however, AR suppresses proliferation of basal cells, supports survival of luminal cells and promotes metastasis (Phé et al., 2010).

When PCa progresses to castration resistance, AR activation and signaling remains sustained through a variety of mechanisms. Several molecular mechanisms have been described for the ability of $\mathrm{AR}$ to retain signaling activity in castration resistant $\mathrm{PCa}$. These mechanisms include the amplification of AR gene copy number in approximately one-third of castration-resistant carcinomas (Tomlins et al., 2005). Another 10-30\% of tumors have gain-of-function mutations of AR that may confer increased protein stability, greater sensitivity to androgens, novel responses to other steroid hormones, ligand-independent activity, or increased recruitment of AR coactivator proteins (Demichelis et al., 2007). In addition, recent studies have shown that expression of alternative splice isoforms encoding constitutively active AR variants also occurs in castration-resistant cancer (Maher et al., 2009). Finally, an unusual mechanism for increased AR signaling activity is the endogenous expression of androgen synthetic enzymes by tumor tissue, which can lead to de novo androgen synthesis or conversion of weaker adrenal androgens into testosterone and dihydrotestosterone (Varambally et al., 2005). Ligand-independent activation of AR activity can 
also take place through activation of growth factor signaling pathways. Notably, up-regulation of the PI3K pathway through Pten deletion appears to be particularly effective, as PIN lesions in Nkx3.1; Pten double-mutant mice display castration resistance prior to carcinoma formation (Tomlins et al., 2007).

\section{Conclusion}

There is an urgent need for novel biomarkers for assessing $\mathrm{CaP}$ diagnosis and prognosis, due to the highly variable natural history of CaP. "Traditional" markers cannot reliably distingish the potientially life-threatenig cancer from insignificant cancer. The development of molecular and immunohistochemical methods enabled the identification of potential biomarkers in relation to prognosis. Numerous promising markers and approaches have been identified and used (expression of FOXA1, loss of PTEN, fusion of genes TMPRSS2 ERG and ETV1, C-MYC, ANXA3, AR and FOXA1, AMACR, GWAS, epigenetic modifications, next generation sequencing, combination of "traditional" markers with novel biomarkers). In addition, attempts to identify cancers with different response to hormonal therapy have been used. The common feature of most current studies is their lack of prospectivity, limited mumber of patients and have to be verified in larger prospective studies. However, some of these markers may be translated into clinical practice in future and can help to assess prognosis of $\mathrm{CaP}$ more accurately.

\section{Acknowledgement}

This study was supported by the grant VEGA No. $1 / 1250 / 12$.

\section{Author's Contributions}

All authors equally contributed in this work.

\section{Ethics}

This article is original and contains unpublished material. The corresponding author confirms that all of the other authors have read and approved the manuscript and no ethical issues involved.

\section{References}

Ahmad, I., R. Patel, L.B. Singh, C. Nixon and M. Seywright et al., 2011. HER2 overcomes PTEN (loss)-induced senescence to cause aggressive prostate cancer. Proceedings of the National Academy of Sciences of the United States of America, Sep. 19-27, pp: 6392-6397.

DOI: $10.1073 /$ pnas. 1101263108
Augello, M.A., T.E. Hickey and K.E. Knudsen, 2011. FOXA1: Master of steroid receptor function in cancer. EMBO J., 30: 3885-3894. PMID: 21934649

Beuten, J., J.A. Gelfond, M.L. Martinez-Fierro, K.S. Weldon and A.C. Crandall et al., 2009. Association of chromosome $8 \mathrm{q}$ variants with prostate cancer risk in Caucasian and Hispanic men. Carcinogenesis, 30: 1372-1379. PMID: 19528667

Bhatia, E., G. Choudhuri, S.S. Sikora, O. Landt and A. Kage et al., 2002. Tropical calcific pancreatitis: Strong association with SPINK1 trypsin inhibitor mutations. Gastroenterology, 123: 1020-1025. PMID: 12360463

Chen, H., S.W. Tu and J.T. Hsieh, 2005. Downregulation of human DAB2IP gene expression mediated by polycomb Ezh2 complex and histone deacetylase in prostate cancer. J. Biol. Chem., 280: 22437-22444. PMID: 15817459

Cibull, T.L., T.D. Jones, L. Li, J.N. Eble and L. Ann Baldridge et al., 2006. Overexpression of Pim-1 during progression of prostatic adenocarcinoma. J. Clin. Pathol., 59: 285-288. PMID: 16505280

Clarke, R.A., H.J. Schirra, J.W. Catto, M.F. Lavin and R.A. Gardiner, 2010. Markers for detection of prostate cancer. Cancers, 2: 1125-1154.

DOI: $10.3390 /$ cancers2021125

Cornu, J.N., G. Cancel-Tassin, C. Egrot, C. Gaffory and F. Haab et al., 2013. Urine TMPRSS2: ERG fusion transcript integrated with PCA3 score, genotyping and biological features are correlated to the results of prostatic biopsies in men at risk of prostate cancer. Prostate, 73: 242-249. DOI: 10.1002/pros.22563

Dang, C.V., K.A. O'Donnell, K.I. Zeller, T. Nguyen and R.C. Osthus et al., 2006. The c-Myc target gene network. Semin Cancer Biol., 16: 253-264. DOI: 10.1016/j.semcancer.2006.07.014

Demichelis, F., K. Fall, S. Perner, O. Andrén and F. Schmidt et al., 2007. TMPRSS2: ERG gene fusion associated with lethal prostate cancer in a watchful waiting cohort. Oncogene, 26: 4596-4599. DOI: 10.1038/sj.onc. 1210237

Ding, Z., C.J. Wu, G.C. Chu, Y. Xiao and D. Ho et al., 2011. SMAD4-dependent barrier constrains prostate cancer growth and metastatic progression. Nature, 470: 269-273. DOI: 10.1038/nature09677

Ellwood-Yen, K., T.G. Graeber, J. Wongvipat, M.L. Iruela-Arispe and J. Zhang et al., 2003. Mycdriven murine prostate cancer shares molecular features with human prostate tumors. Cancer Cell, 4: 223-238. PMID: 14522256

Epstein, J.I., M. Amin, L. Boccon-Gibod, L. Egevad and P.A. Humphrey et al., 2005. Prognostic factors and reporting of prostate carcinoma in radical prostatectomy and pelvic lymphadenectomy specimens. Scand J. Urol. Nephrol. Suppl, PMID: 16019758 
Febbo, P.G., 2009. Genomic approaches to outcome prediction in prostate cancer. Cancer, 115: 3046-3057. DOI: $10.1002 /$ cncr. 24350

Festuccia, C., G.L. Gravina, A. Mancini, P. Muzi and E.D. Cesare et al., 2014. Trifluoroibuprofen inhibits $\alpha$-Methylacyl Coenzyme A Racemase (AMACR/P504S), Reduces cancer cell proliferation and inhibits in vivo tumor growth in aggressive prostate cancer models. Anticancer Agents Med. Chem., 14: 1031-1041. PMID: 24712326

Flavin, R.J., A. Pettersson, W.K. Hendrickson, M. Fiorentino and S.P. Finn et al., 2014. SPINK1 protein expression and prostate cancer progression. Clin. Cancer Res., DOI: 10.1158/1078-0432.CCR-13-1341

Gerke, V., C.E. Creutz and S.E. Moss, 2005. Annexins: Linking $\mathrm{Ca} 2+$ signalling to membrane dynamics. Nat. Rev. Mol. Cell Biol., 6: 449-461. PMID: 15928709

Halvorsen, O.J., S.A. Haukaas and L.A. Akslen, 2003. Combined loss of PTEN and p27 expression is associated with tumor cell proliferation by Ki-67 and increased risk of recurrent disease in localized prostate cancer. Clin. Cancer Res., 9: 1474-1479. PMID: 12684422

Harden, S.V., Z. Guo, J.I. Epstein and D. Sidransky, 2003. Quantitative GSTP1 methylation clearly distinguishes benign prostatic tissue and limited prostate adenocarcinoma. J. Urol., 169: 1138-1142. DOI: 10.1097/01.ju.0000049627.90307.4d

Hartwell, L., D. Mankoff, A. Paulovich, S. Ramsey and E. Swisher, 2006. Cancer biomarkers: A systems approach. Nat. Biotechnol., 24: 905-908. Doi: $10.1038 /$ nbt0806-905

Hassan, M.I., V. Kumar, T.P. Singh and S. Yadav, 2008. Purification and characterization of zinc alpha2-glycoprotein-prolactin inducible protein complex from human seminal plasma. J. Sep. Sci., 31: 2318-2324. DOI: 10.1002/jssc. 200700686

Henshall, S.M., L.G. Horvath, D.I. Quinn, S.A. Eggleton and J.J. Grygiel et al., 2006. Zinc-alpha2glycoprotein expression as a predictor of metastatic prostate cancer following radical prostatectomy. J. Natl. Cancer Inst., 98: 1420-1424. DOI: $10.1093 /$ jnci/djj378

Hessels, D., F.P. Smit, G.W. Verhaegh, J.A. Witjes and E.B. Cornel et al., 2007. Detection of TMPRSS2ERG fusion transcripts and prostate cancer antigen 3 in urinary sediments may improve diagnosis of prostate cancer. Clin. Cancer Res., 13: 5103-5108. DOI: 10.1158/1078-0432.CCR-07-0700

Jain, R.K., R.J. Mehta, H. Nakshatri, M.T. Idrees and S.S. Badve, 2011. High-level expression of forkhead-box protein A1 in metastatic prostate cancer. Histopathology, 58: 766-772. DOI: 10.1111/j.1365-2559.2011.03796.x
Jemal, A., R. Siegel, E. Ward, Y. Hao and J. Xu et al., 2009. Cancer statistics, 2009. CA Cancer J. Clin., 59: 225-249. DOI: 10.3322/caac. 20006

Jiang, W., B. Freidlin and R. Simon, 2007. Biomarker-adaptive threshold design: A procedure for evaluating treatment with possible biomarker-defined subset effect. J. Natl. Cancer Inst., 99: 1036-1043. DOI: 10.1093/jnci/djm022

Jiang, Z., C.L. Wu, B.A. Woda, K.A. Iczkowski and P.G. Chu et al., 2004. Alpha-methylacyl-CoA racemase: a multi-institutional study of a new prostate cancer marker. Histopathology, 45: 218-225. DOI: $10.1111 / \mathrm{j} .1365-2559.2004 .01930 . \mathrm{x}$

Katafigiotis, I., S.I. Tyritzis, K.G. Stravodimos, C. Alamanis and K. Pavlakis et al., 2012. Zinc $\alpha 2-$ glycoprotein as a potential novel urine biomarker for the early diagnosis of prostate cancer. BJU Int., 110: E688-693. DOI: 10.1111/j.1464-410X.2012.11501.X

Kawamoto, K., S.T. Okino, R.F. Place, S. Urakami and H. Hirata et al., 2007. Epigenetic modifications of RASSF1A gene through chromatin remodeling in prostate cancer. Clin. Cancer Res., 13: 2541-2548. DOI: 10.1158/1078-0432.CCR-06-2225

Köllermann, J., T. Schlomm, H. Bang, G.P. Schwall and C. Von Eichel-Streiber et al., 2008. Expression and prognostic relevance of annexin A3 in prostate cancer. Eur. Urol., 54: 1314-1323. DOI: $10.1016 /$ j.eururo.2008.01.001

Lapointe, J., S. Malhotra, J.P. Higgins, E. Bair and M. Thompson et al., 2008. hCAP-D3 expression marks a prostate cancer subtype with favorable clinical behavior and androgen signaling signature. Am. J. Surg. Pathol., 32: 205-209. DOI: $10.1097 /$ PAS.0b013e318124a865

Laxman, B., D.S. Morris, J. Yu, J. Siddiqui and J. Cao et al., 2008. A first-generation multiplex biomarker analysis of urine for the early detection of prostate cancer. Cancer Res., 68: 645-649. DOI: 10.1158/0008-5472.CAN-07-3224

Lee, W.H., R.A. Morton, J.I. Epstein, J.D. Brooks and P.A. Campbell et al., 1994. Cytidine methylation of regulatory sequences near the pi-class glutathione Stransferase gene accompanies human prostatic carcinogenesis. Proc. Natl. Acad. Sci. USA, 91: 11733-11737. PMID: 7972132

Leman, E.S., G.W. Cannon, B.J. Trock, L.J. Sokoll and D.W. Chan et al., 2007. EPCA-2: A highly specific serum marker for prostate cancer. Urology, 69: 714-720. DOI: 10.1016/j.urology.2007.01.097

Li, Y., J. Su, X. DingZhang, J. Zhang, M. Yoshimoto and S. Liu et al., 2011. PTEN deletion and heme oxygenase-1 overexpression cooperate in prostate cancer progression and are associated with adverse clinical outcome. J. Pathol., 224: 90-100. DOI: $10.1002 /$ path.2855 
Linja, M.J., K.J. Savinainen, O.R. Saramäki, T.L. Tammela and R.L. Vessella et al., 2001. Amplification and overexpression of androgen receptor gene in hormone-refractory prostate cancer. Cancer Res., 61: 3550-3555. PMID: 11325816

Macconaill, L.E. and L.A. Garraway, 2010. Clinical implications of the cancer genome. J. Clin. Oncol., 28: 5219-5228. DOI: 10.1200/JCO.2009.27.4944

Maher, C.A., C. Kumar-Sinha, X. Cao, S. KalyanaSundaram and B. Han et al., 2009. Transcriptome sequencing to detect gene fusions in cancer. Nature, 458: 97-101. DOI: $10.1038 /$ nature 07638

Manolio, T.A., 2010. Genomewide association studies and assessment of the risk of disease. N Eng. J. Med., 363: 166-176. DOI: 10.1056/NEJMra0905980

Marks, L.S., Y. Fradet, I.L. Deras, A. Blasé and J. Mathis et al., 2007. PCA3 molecular urine assay for prostate cancer in men undergoing repeat biopsy. Urology, 69: 532-535. PMID: 17382159

McMenamin, M.E., P. Soung, S. Perera, I. Kaplan and M. Loda et al., 1999. Loss of PTEN expression in paraffin-embedded primary prostate cancer correlates with high Gleason score and advanced stage. Cancer Res., 59: 4291-4296. PMID: 10485474

Mulholland, D.J., L.M. Tran, Y. Li, H. Cai and A. Morim et al., 2011. Cell autonomous role of PTEN in regulating castration-resistant prostate cancer growth. Cancer Cell, 19: 792-804. DOI: 10.1016/j.ccr.2011.05.006

Nelson, W.G., A.M. De Marzo and S. Yegnasubramanian, 2009. Epigenetic alterations in human prostate cancers. Endocrinology, 150: 3991-4002. DOI: 10.1210/en.2009-0573

Phé, V., O. Cussenot and M. Rouprêt, 2010. Methylated genes as potential biomarkers in prostate cancer. BJU Int., 105: 1364-1370. DOI: 10.1111/j.1464-410X.2009.09167.X

Pulukuri, S.M., S. Patibandla, J. Patel, N. Estes and J.S. Rao, 2007. Epigenetic inactivation of the tissue inhibitor of metalloproteinase-2 (TIMP-2) gene in human prostate tumors. Oncogene, 26: 5229-5237. DOI: $10.1038 /$ sj.onc. 1210329

Reid, B.J., X. Li, P.C. Galipeau and T.L. Vaughan, 2010. Barrett's oesophagus and oesophageal adenocarcinoma: Time for a new synthesis. Nat. Rev. Cancer, 10: 87-101. DOI: 10.1038/nrc2773

Repiska, V., M. Miklosi, A. Zummerova, L. Danisovic and J. Hoffmann et al., 2005. Detection of circulating tumor cells in patients with prostate cancer. Biologia, 60: 703-709.

Ribeiro, F.R., R. Henrique, A.T. Martins, C. Jerónimo and M.R. Teixeira, 2007. Relative copy number gain of MYC in diagnostic needle biopsies is an independent prognostic factor for prostate cancer patients. Eur. Urol., 52: 116-125. DOI: $10.1016 /$ j.eururo.2006.09.018
Robbins, C., J.B. Torres, S. Hooker, C. Bonilla and W. Hernandez et al., 2007. Confirmation study of prostate cancer risk variants at $8 \mathrm{q} 24$ in African Americans identifies a novel risk locus. Genome Res., 17: 1717-1722. DOI: 10.1101/gr.6782707

Rogers, C.G., G. Yan, S. Zha, M.L. Gonzalgo and W.B. Isaacs et al., 2004. Prostate cancer detection on urinalysis for alpha methylacyl coenzyme a racemase protein. J. Urol., 172: 1501-1503. PMID: 15371879

Salagierski, M. and J.A. Schalken, 2012. Molecular diagnosis of prostate cancer: PCA3 and TMPRSS2: ERG gene fusion. J. Urol., 187: 795-801. DOI: 10.1016/j.juro.2011.10.133

Salami, S.S., F. Schmidt, B. Laxman, M.M. Regan and D.S. Rickman et al., 2013. Combining urinary detection of TMPRSS2: ERG and PCA3 with serum PSA to predict diagnosis of prostate cancer. Urol. Oncol., 31: 566-571. DOI: 10.1016/j.urolonc.2011.04.001

Sreekumar, A., B. Laxman, D.R. Rhodes, S. Bhagavathula and J. Harwood et al., 2004. Humoral immune response to alpha-methylacyl-CoA racemase and prostate cancer. J. Natl. Cancer Inst., 96: 834-843. DOI: 10.1093/jnci/djh145

Thomas, G.V., S. Horvathm, B.L. Smith, K. Crosby and L.A. Lebel et al., 2004. Antibody-based profiling of the phosphoinositide 3-kinase pathway in clinical prostate cancer. Clin. Cancer Res., 10: 8351-8356. DOI: 10.1158/1078-0432.CCR-04-0130

Tomlins, S.A., D.R. Rhodes, S. Perner, S.M. Dhanasekaran and R. Mehra et al., 2005. Recurrent fusion of TMPRSS2 and ETS transcription factor genes in prostate cancer. Science, 310: 644-648. PMID: 16254181

Tomlins, S.A., M.A. Rubin and A.M. Chinnaiyan, 2006. Integrative biology of prostate cancer progression. Annu. Rev. Pathol., 1: 243-271. DOI: 10.1146/annurev.pathol.1.110304.100047

Tomlins, S.A., R. Mehra, D.R. Rhodes, X. Cao and L. Wang et al., 2007. Integrative molecular concept modeling of prostate cancer progression. Nat. Genet., 39: 41-51. DOI: 10.1038/ng1935

Tosoian, J.J., S. Loeb, A. Kettermann, P. Landis and D.J. Elliot et al., 2010. Accuracy of PCA3 measurement in predicting short-term biopsy progression in an active surveillance program. J. Urol., 183: 534-538. DOI: 10.1016/j.juro.2009.10.003

Van Gils, M.P., D. Hessels, O. Van Hooij, S.A. Jannink and W.P. Peelen et al., 2007. The timeresolved fluorescence-based PCA3 test on urinary sediments after digital rectal examination; a Dutch multicenter validation of the diagnostic performance. Clin. Cancer Res., 13: 939-943. DOI: 10.1158/1078-0432.CCR-06-2679 
Varambally, S., J. Yu, B. Laxman, D.R. Rhodes and R. Mehra et al., 2005. Integrative genomic and proteomic analysis of prostate cancer reveals signatures of metastatic progression. Cancer Cell, 8: 393-406. DOI: 10.1016/j.ccr.2005.10.001

Varambally, S., S.M. Dhanasekaran, M. Zhou, T.R. Barrette and C. Kumar-Sinha et al., 2002. The polycomb group protein EZH2 is involved in progression of prostate cancer. Nature, 419: 624-249. DOI: $10.1038 /$ nature 01075

Vivanco, I. and C.L. Sawyers, 2002. The phosphatidylinositol 3-Kinase AKT pathway in human cancer. Nat. Rev. Cancer, 2: 489-501. PMID: 12094235

Witt, H., W. Luck, H.C. Hennies, M. Classen and A. Kage et al., 2000. Mutations in the gene encoding the serine protease inhibitor, Kazal type 1 are associated with chronic pancreatitis. Nat. Genet, 25: 213-216. DOI: $10.1038 / 76088$

Yegnasubramanian, S., J. Kowalski, M.L. Gonzalgo, M. Zahurak and S. Piantadosi et al., 2004. Hypermethylation of $\mathrm{CpG}$ islands in primary and metastatic human prostate cancer. Cancer Res., 64: 1975-1986. DOI: 10.1158/0008-5472.CAN-03-3972

Zha, S., S. Ferdinandusse, S. Denis, R.J. Wanders and C.M. Ewing et al., 2003. Alpha-methylacyl-CoA racemase as an androgen-independent growth modifier in prostate cancer. Cancer Res., 63: 7365-7376. PMID: 14612535
Zheng, S.L., J. Sun, F. Wiklund, S. Smith and P. Stattin et al., 2008. Cumulative association of five genetic variants with prostate cancer. N Engl. J. Med., 358: 910-919. DOI: 10.1056/NEJMoa075819

Ziaran, S., F.M. Goncalves and J.S. Breza, 2011. Bone mineral density, pathological fractures and bisphosphonate therapy in prostate cancer patients on androgen deprivation therapy. Endocr. Regul., 45: 199-204. PMID: 22073949

Ziaran, S., F.M. Goncalves and J.S. Breza, 2013a. Patients with prostate cancer treated by ADT have significantly higher fibrinogenemia than healthy control. World J. Urol., 31: 289-292. DOI: 10.1007/s00345-012-0926-X

Ziaran, S., F.M. Goncalves and J.S. Breza, 2013b. Complex metabolic and skeletal changes in men taking longterm androgen deprivation therapy. Clin. Genitourin Cancer, 11: 33-38. DOI: 10.1016/j.clgc.2012.08.005

Ziaran, S., F.M. Goncalves, J. Wendl, B. Trebaticky and J.S. Breza, 2009. Evaluation of bone mass density on patients with prostate cancer prior to the start of androgen deprivation therapy. Bratisl. Lek Listy, 110: 559-562. PMID: 19827339

Zummerova, A., P. Labas, D. Bohmer, M. Blasko and S. Polak et al., 2010. The application of the RTPCR method for the staging of the prostate cancer progression. Gen. Physiol. Biophys., 29: 362-372. DOI: $10.4149 / g p b \_2010 \_04 \_362$ 